\title{
Formulation and Evaluation of Microspheres Based on Gelatin-Mucin Admixtures for the Rectal Delivery of Cefuroxime Sodium
}

\author{
KC Ofokansi ${ }^{*}$ and M U Adikwu
}

Drug Delivery Research Unit, Department of Pharmaceutics, University of Nigeria, Nsukka, Enugu State, Nigeria

\begin{abstract}
Purpose: Swellable microspheres based on polymers or their admixtures are frequently employed as drug delivery systems to achieve a controlled release and site-specific targeting of the incorporated drug. The objective of the present study was to enhance the rectal delivery of cefuroxime sodium by entrapping it into water-swellable gelatin-mucin microspheres.

Method: Cefuroxime sodium-loaded microspheres containing admixtures of gelatin and porcine mucin were prepared via an emulsification-crosslinking technique. The drug entrapment efficiency of the microspheres was evaluated in citrate/phosphate buffer $(\mathrm{pH} 7.4)$ while the swelling properties was evaluated in both simulated gastric fluid (SGF) without pepsin and simulated intestinal fluid (SIF) without pancreatin ( $\mathrm{pH}$ 1.2). Release of cefuroxime sodium from the microspheres was evaluated in vitro in SIF and further evaluated in vivo after rectal administration to male Wistar rats.

Result: Results obtained showed that a high entrapment efficiency, most notably manifested in microspheres formulated with equal portions of gelatin and mucin, led to a high release (up to $85 \%$ ) and also a high bioavailability of the incorporated drug. Formulations based on varying portions of gelatin and mucin also showed high drug loading efficiency which also resulted in high drug release in SIF within $3 \mathrm{~h}$. Drug release from the different formulations was observed to be rapid and generally showed a biphasic pattern. The mean AUC was shown to be formulation-dependent with values of $168 \pm 1.93 \mu \mathrm{g} . \mathrm{h} / \mathrm{ml}$ for the control, $262 \pm 3.47 \mu \mathrm{g} . \mathrm{h} / \mathrm{ml}$ for microspheres based on gelatine only and $328 \pm 2.55 \mu \mathrm{g} . \mathrm{h} / \mathrm{ml}$ for microspheres formulated with equal parts of gelatin and mucin.

Conclusion: The inclusion of S-mucin in the composition of the microspheres has an enhancer effect on the release and rectal bioavailability of cefuroxime sodium which may be exploited in the design of a rectal delivery system of the drug.
\end{abstract}

Keywords: Gelatin-mucin microspheres, cefuroxime sodium, rectal bioavailability.

*Corresponding Author: E-mail: Kcofokansi@yahoo.com Phone: +234-80-37794873 


\section{INTRODUCTION}

The rectal route has been frequently exploited for the systemic delivery of drugs in situations where it is desirable to avoid hepatic first-pass metabolism $^{1}$ or to prolong drug release ${ }^{2}$. For effective retention of medication on the rectal mucosa, the drug delivery system should display a mucoadhesive property.

Microspheres have been extensively studied for use as drug delivery systems, where they have been shown to protect sensitive macromolecules from enzymatic and acid degradation, and allow controlled release and tissue targeting of the formulated drug ${ }^{3-13}$. Microspheres prepared with gelatin as the polymer have been found to be highly mucoadhesive and have been used for the controlled release of many drugs ${ }^{14}$. Microspheres prepared from admixtures of gelatin and crosslinked chitosan demonstrated some advantage over that prepared from gelatin alone in terms of better controlled release rate of cimetidine ${ }^{14}$. The reported ionic interaction of mucin with many biologically important compounds including polymers ${ }^{15}$ suggested that mucoadhesive microspheres could be produced from admixtures of mucin and gelatin. The parenteral route of administration of antibiotics (including cefuroxime sodium) is fraught with myriad of draw-backs especially pain at the injection site which often leads to poor patient compliance or complete non-compliance with its obvious consequences. The rectal route of administration of cefuroxime sodium could, therefore, offer better advantages in terms of enhanced patient compliance and could also be a potential alternative to the parenteral route for the delivery of cefuroxime sodium.

The search for alternative routes of delivery of many drugs including insulin and other peptides is continuing; hence our current efforts to explore the rectal route for the delivery of cefuroxime sodium, an acid labile cephalosporin. The objective of this study was to develop mucoadhesive formulations (microspheres) based on gelatin and its admixtures with porcine mucin with a view to enhancing the rectal delivery of cefuroxime sodium through entrapment into these microspheres. This way, a viable alternative route of delivery of cefuroxime sodium, in addition to the parenteral route, would have been provided.

\section{MATERIALS AND METHODS Materials}

Cefuroxime sodium powder (GlaxoWellcome, England); citric acid, sodium hydroxide (Merck, Germany); acetone, concentrated $\mathrm{HCl}$, glutaraldehyde, disodium hydrogen phosphate, sodium chloride (BDH, England); type A gelatin (75 bloom), monobasic potassium phosphate (Sigma Chemical Co., USA) were used as procured from the manufacturers without further purification. All other reagents were analytical grade and used as such. Distilled water was obtained from an all-glass still. The animal experimental protocols were approved by our institution's Animal Ethics Committee.

\section{Isolation of the porcine mucin}

The small intestines of freshly slaughtered pigs (within $1 \mathrm{~h}$ post mortem) were obtained from the abattoir of the Animal Science Department in our University and dissected, starting from the jejunum to the ileocaecal sphincter. The intestines, sectioned into short lengths, were flushed through with chilled saline, and the mucosal surface was exposed by longitudinal dissection. By using a microscope slide, the mucus layer was gently scraped off and diluted with four times its volume of distilled water. The gel was homogenized for $2 \mathrm{~h}$ at $4{ }^{\circ} \mathrm{C}$ and thereafter exhaustively dialysed against distilled water using a $12 \mathrm{KDa}$ molecular weight cut-off (MWCO) dialysis membrane. The dialysate was finally centrifuged at $10,000 \mathrm{rpm}$ for $30 \mathrm{~min}$ to yield a supernatant of water-soluble mucus glycoprotein layers and lower layer of insoluble mucus glycoprotein. The supernatants were collected separately, pooled and lyophilized at $40^{\circ} \mathrm{C}$ for $48 \mathrm{~h}$ to obtain flakes of soluble (S) mucin, which were powdered and used for the study.

\section{Preparation of gelatin-mucin admixtures}

$1 \mathrm{~g}$ quantity of gelatin was dispersed in $50 \mathrm{ml}$ of citrate/phosphate buffer of $\mathrm{pH} 3.4$. An equal amount of S-mucin was similarly weighed out 
and mixed thoroughly with the dispersion of gelatin in a beaker. The mixture was left to stand for $24 \mathrm{~h}$ in order to attain maximum hydration. It was then homogenized by stirring with a glass rod for $5 \mathrm{~min}$. The procedure was repeated to obtain S-mucin to gelatin in ratios of $1: 1,1: 2,1: 3$, and $1: 4$.

\section{Preparation of the gelatin-mucin microspheres}

A dispersion of each gelatin-mucin admixture (25 $\% \mathrm{v} / \mathrm{v}$ in arachis oil) was used in the preparation of the microspheres. $1 \mathrm{~g}$ of cefuroxime sodium powder was dispersed in $25 \mathrm{ml}$ of the gelatinmucin dispersion and heated until the temperature of the dispersion was brought to 40 ${ }^{\circ} \mathrm{C}$. The dispersion was further extruded dropwise, with the aid of a syringe into pure arachis oil maintained at $40{ }^{\circ} \mathrm{C}$ on a thermostatically controlled hot plate-magnetic stirrer. The mixture was stirred at a speed of $500 \mathrm{rpm}$ for $30 \mathrm{~min}$. Glutaraldehyde was added to a concentration of about $1 \% \mathrm{v} / \mathrm{v}$ to cross-link the microspheres in situ and stirred further for 30 min. The resulting mixture was centrifuged at $6000 \mathrm{rpm}$ for $10 \mathrm{~min}$ to collect the microspheres. The microspheres collected were washed with acetone to remove excess oil and then dried at the ambient temperature of $28 \pm 2{ }^{\circ} \mathrm{C}$.

\section{Determination of entrapment efficiency of the microspheres}

A quantity $(100 \mathrm{mg})$ of the microspheres was placed in a beaker containing $100 \mathrm{ml}$ of the citrate/phosphate buffer $(\mathrm{pH}$ 7.4) The dispersion was vortexed repeatedly to break up the microspheres and cause them to discharge their drug contents completely. The solution was then filtered and analyzed spectrophotometrically at a wavelength of $280 \mathrm{~nm}$ using a UV-Vis spectrophotometer (Spectronic 21D). The drug concentration in each batch of the microspheres was calculated from a Beer's plot previously determined for cefuroxime sodium. An average of five determinations was taken as the mean drug content for each batch of microspheres.

\section{In vitro drug release studies}

The USP XXVII paddle method was adopted in this study. The release medium consisted of
$500 \mathrm{ml}$ of freshly prepared SIF (pH 7.4) maintained at $37 \pm 1^{\circ} \mathrm{C}$. SIF was selected as the release medium since it has a $\mathrm{pH}$ very close to that of the rectal fluids. A known quantity (100 $\mathrm{mg}$ ) of each batch of the microspheres was placed in the appropriate chamber of the release apparatus and agitated at $100 \mathrm{rpm}$. At predetermined time intervals, $1 \mathrm{ml}$ aliquots of the release medium were withdrawn, appropriately diluted and assayed spectrophotometrically at $280 \mathrm{~nm}$. At every interval, $1 \mathrm{ml}$ of fresh SIF was added to replace the sample that was withdrawn. The concentrations of the withdrawn samples were calculated with reference to the standard Beer's plot. Four replicate release studies were performed in each case and the mean values were taken.

\section{Pharmacokinetic studies}

Male Wistar rats aged two months with a mean weight of $200 \pm 10.5 \mathrm{~g}$ were obtained from the Department of Veterinary Pathology and Microbiology, of our University and used for the study. The rats were allowed to acclimatize to the new environmental conditions of our laboratories for one week before use. Three groups of eight animals each were used for the study. An amount of the microspheres equivalent to a dose of $100 \mathrm{mg}$ of the $\mathrm{drug} / \mathrm{kg}$ body weight of the rats was carefully transferred into the empty bodies of capsule no. 3. A positive control was set up by enclosing an equal amount of the pure cefuroxime sodium powder equivalent to that in the microspheres. By means of the capsules the drug was administered rectally to the rats. At regular time intervals of $30 \mathrm{~min}$ for the first one hour, and then subsequently at $1 \mathrm{~h}$ intervals, $0.5 \mathrm{ml}$ of blood samples were withdrawn from the orbital sinus of the rat ${ }^{16,17}$.

\section{Analysis of cefuroxime sodium in protein- free rat plasma}

The method of Tietz ${ }^{18}$ was adopted to prepare a protein-free filtrate. The blood sample $(0.2 \mathrm{ml})$ was added to a test-tube containing $1.8 \mathrm{ml}$ of 3 $\%$ trichloroacetic acid (TCA). The test-tube was shaken gently to ensure proper mixing and allowed to stand for 5-10 $\mathrm{min}$. The test-tube was further centrifuged for $10 \mathrm{~min}$ at $3000 \mathrm{rpm}$ 
Table 1: Release kinetic parameters of cefuroxime sodium from the microspheres in SIF

\begin{tabular}{cccc}
\hline \hline $\begin{array}{c}\text { Microsphere } \\
\text { (mucin- }\end{array}$ & \multicolumn{3}{c}{ Release parameters } \\
\cline { 2 - 4 } gelatin ratio) & $\mathrm{n}$ & $\mathrm{K}$ & $\mathrm{r}$ \\
\hline $1: 1$ & 0.50 & 0.1931 & 0.9965 \\
$1: 2$ & 0.48 & 0.1983 & 0.9881 \\
$1: 3$ & 0.96 & 0.2214 & 0.9915 \\
$1: 4$ & 0.82 & 0.2651 & 0.9853 \\
Gelatin & 0.57 & 0.3273 & 0.9855 \\
alone & & &
\end{tabular}

$\bar{n}=$ Release exponent; $K=$ Release kinetic constant; $r=$ Correlation coefficient

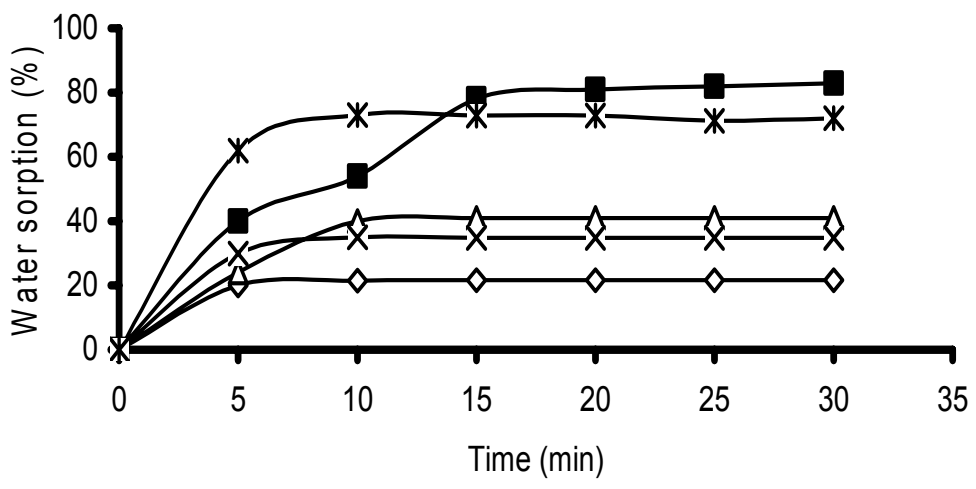

Fig.1 : Swelling profiles of the microspheres in SGF

$\checkmark$ Gelatin alone $\neg-1: 1 \rightarrow-1: 2-X-1: 3-x-1: 4$

after which $1 \mathrm{ml}$ of the clear supernatant layer was collected and analyzed spectrophotometrically without dilution at 280 $\mathrm{nm}$. The blank was a $3 \%$ solution of TCA. An absorption spectrum previously constructed for a solution of cefuroxime sodium in TCA did not show any shift from the earlier wavelength of maximum absorption; an indication that no significant interaction occurred between the two compounds (i.e. TCA and cefuroxime sodium).

\section{Statistical data analysis}

Statistical data analysis were performed using the student's t-test with $p \leq 0.05$ as the minimal level of significance.

\section{RESULTS}

The results of the water sorption studies carried out in two different media(SIF and SGF) are shown in Figs. 1 and 2 . It can be seen from these figures that microspheres prepared from admixtures of gelatin and S-mucin showed higher swelling tendency especially in SGF where between 35 and $82 \%$ water sorption was recorded when compared to that prepared from gelatin only which showed a water sorption of 25 $\%$. Water sorption for all the batches of microspheres including that formulated with gelatin only was quite high in SIF and ranged between 150 and $290 \%$. The highest water sorption capacity was shown by microspheres formulated with equal portions of gelatin and mucin and this was followed closely by 


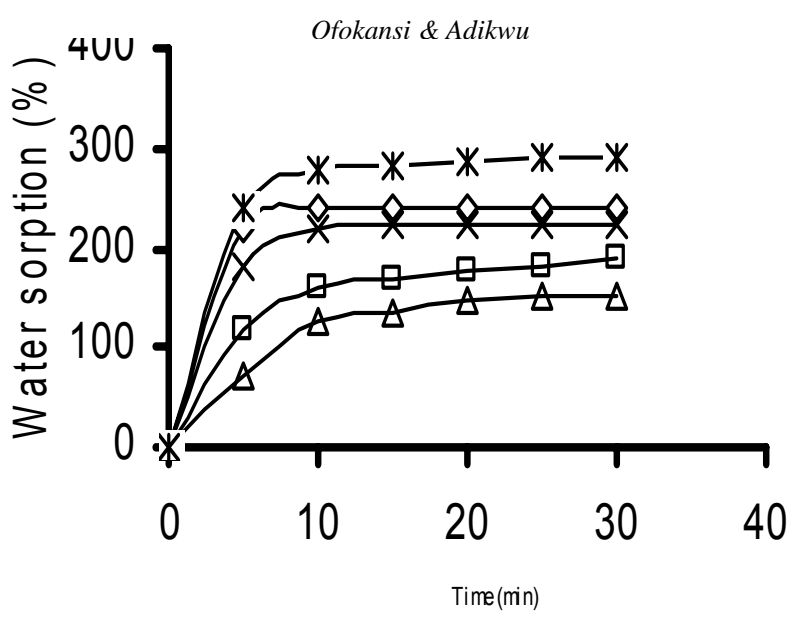

Fig. 2: Swelling profiles of the microspheresin SIF

- Gelatin alone $\rightarrow 1: 1 \rightarrow 1: 2 \rightarrow 1: 3 \quad$

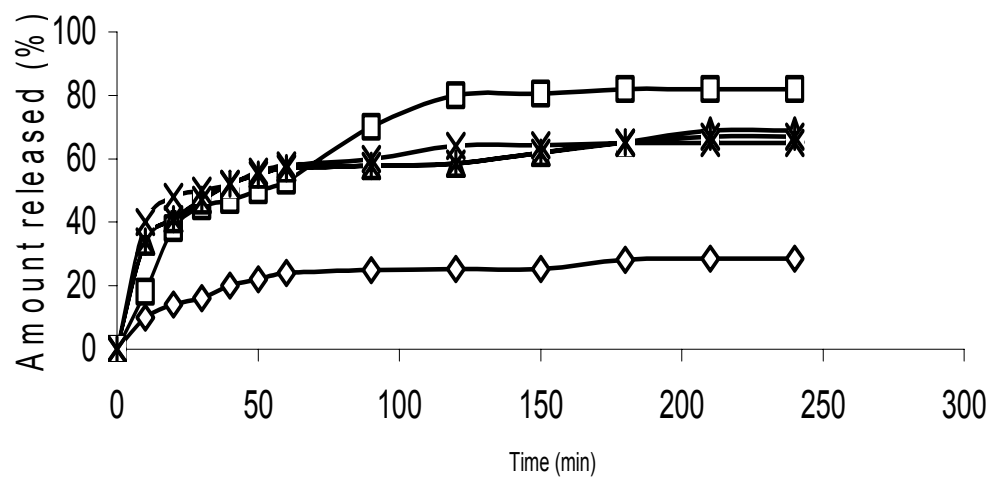

Fig. 3: Release profile of cefuroxime sodium from the microspheres in SIF $\neg-1: 2-\square-1: 1 \rightarrow-$ Gelatin alone $-x-1: 3 \rightarrow-1: 4$

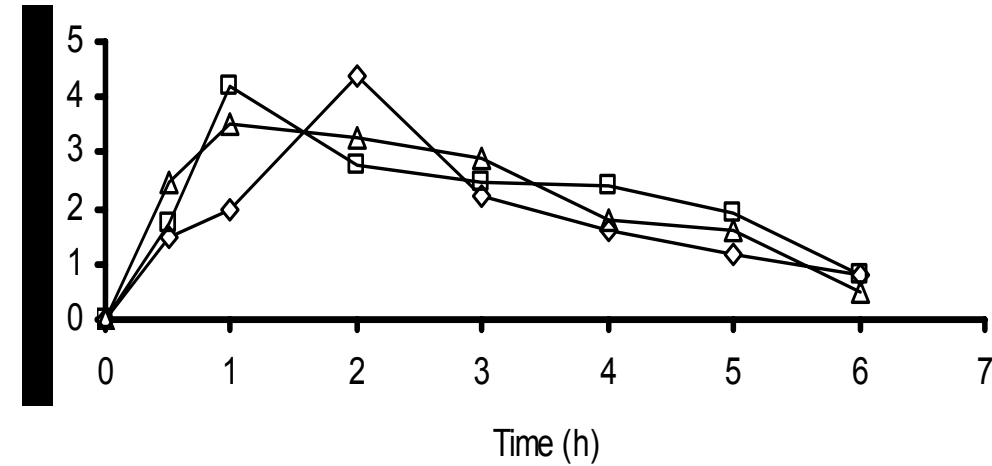

Fig. 4: Plasma cefuroxime sodium versus time profile for the microspheres

$\neg 1: 1 \rightarrow$ Gelatin alone $\neg$ control 
microspheres based on gelatin only. Water absorption and the rate of water absorption by the microspheres followed the order:1:1>1:2> $1: 3>1: 4>$ gelatin alone in SGF while the order in SIF is; $1: 4>$ gelatin alone $>1: 3>1: 2>1: 1$.

The drug entrapment efficiency was observed to be dependent on the composition of the microspheres. Microspheres prepared from combinations of gelatin and S-mucin entrapped greater amounts $(p \leq 0.05)$ of cefuroxime sodium in comparison with those prepared from gelatin alone. The drug entrapment efficiency of all batches of the microspheres was in the range of 72.6 to $98.4 \%$ with microspheres formulated with gelatin-mucin (1:1) recording the highest drug entrapment. The general pattern was that drug entrapment decreased with increasing proportions of gelatin in the gelatin-mucin admixtures used in preparing the microspheres.

The release profile of cefuroxime sodium from the microspheres in SIF is shown in Fig. 3. There was an initial rapid release of cefuroxime sodium from the microspheres in SIF within 30 min and this was followed by a much slower release over the next $150 \mathrm{~min}$. Drug release was highest from microspheres prepared with equal proportions of S-mucin and gelatin (1:1) where up to $85 \%$ of the drug was released within $3 \mathrm{~h}$. A characteristic feature of the release profile of cefuroxime sodium from the microspheres in SIF is the biphasic pattern of release.

The plasma concentration versus time curves for cefuroxime sodium, after rectal administration to male Wistar rats, are depicted in Fig. 4. The areas under the plasma level versus time curves (AUC) were evaluated using the trapezoid rule based on a non-compartmental pharmacokinetic analysis. The pharmacokinetic parameters as can be seen from Fig. 4 showed a higher peak plasma concentration of $4.5 \pm 0.39 \mu \mathrm{g} / \mathrm{ml}$ for microspheres based on gelatin-mucin in the ratio $1: 1$ when compared to that for the control $(3.5 \pm 0.45 \mu \mathrm{g} / \mathrm{ml})$ or the microspheres based on gelatin only $(4.0 \pm 0.45 \mu \mathrm{g} / \mathrm{ml})$. The mean AUC was shown to be dependent on the formulation with values of $168 \pm 1.93 \mu \mathrm{g} . \mathrm{h} / \mathrm{ml}$ for the control , $262 \pm 3.47 \mu \mathrm{g} . \mathrm{h} / \mathrm{ml}$ for microspheres based on gelatin only and $328 \pm 2.55 \mu \mathrm{g} . \mathrm{h} / \mathrm{ml}$ for microspheres formulated with gelatin and mucin in the ratio 1:1 which gives an indication that the inclusion of mucin in the microsphere formulation enhanced the rectal bioavailability of cefuroxime sodium.

\section{DISCUSSION}

The water sorption behaviour in the two media may be an indication of a probable modification of the gelatin microspheres by the mucin. The higher amount and rate of water absorption in SIF than in SGF may also be attributable to the type of gelatin (type A) used in preparing the microspheres. This type of gelatin is known to be produced from acidic precursors and would, therefore, be expected to swell more in an alkaline environment of the SIF than in SGF.

The wide variation in the drug contents of the different batches of the microspheres could be a consequence of the varying degrees of drug sedimentation and the relative partitioning of cefuroxime sodium between the dispersed and continuous phases of the emulsion prior to cross-linking of the polymer admixtures.

The rapid release in the first $30 \mathrm{~min}$ as is evident in Fig. 3 is possibly due to a burst effect caused by the leaching out of the unentrapped drug adhering to the surface of the microspheres after the initial rapid hydration and swelling. The high and rapid release of cefuroxime sodium from the microspheres in SIF, in addition to the burst effect, may also may be a result of the high rate of hydration and swelling of the microspheres in SIF, which, in turn, could be, attributable to the type of gelatin (type A) used in preparing the microspheres. The subsequent slow release phase could be a consequence of the decreasing residual amount of drug in the microspheres and the build-up of drug concentration in the dissolution medium in the course of time. This indicates that once the drug adhering to the microspheric surface has leached, the drug release obeys the Higuchi membrane diffusion controlled model ${ }^{19}$

Further analysis of the release of cefuroxime sodium from the microspheres was done using 
the Fickian diffusion model ${ }^{20}$ to determine the mechanism of release. To understand the release mechanism of cefuroxime sodium from the microspheres, the release rate was described with the following equations ${ }^{20}$ :

$$
\frac{M_{t}}{M}=K^{n}
$$

$$
\log \frac{M_{t}}{M}=\log K+n \log t
$$

$M_{t} / M$ is the fraction of drug released at time $t, K$ is a kinetic constant that incorporates the structural and geometric characteristics of the release device and $n$ is the release exponent indicative of the mechanism of release. An $n$ value of 1.0 corresponds to zero-order release kinetics (case-II transport). An $\mathrm{n}$ value greater than 0.5 but less than 1.0 means an anomalous (non-Fickian) diffusion release model; $\mathrm{n}=0.5$ indicates Fickian diffusion while $n>1.0$ indicates a super case-Il relaxational release ${ }^{21}$. The kinetic parameters, $\mathrm{n}$ and $\mathrm{K}$ as calculated from plots of $\log M_{t} / M$ versus $\log t$ for cefuroxime sodium are presented in Table 1 where can be seen that the values of the release exponent, $n$, were in the range 0.48 to 0.96 , indicating that the release of cefuroxime sodium from the microspheres in SIF occurred predominantly by diffusion following an initial non-Fickian transport.

Compared with the control and microspheres formulated with gelatin only, it is interesting to note that the bioavailability of cefuroxime sodium via the rectal route was higher (AUC $=328 \mu \mathrm{g} . \mathrm{h}$ $\mathrm{ml}^{-1}$ ) from microspheres formulated with an admixture of S-mucin and gelatin in the ratio 1:1. The in vitro release study in SIF showed that higher amount of cefuroxime sodium was released from the 1:1 mucin-gelatin based microspheres than from gelatin-only based microspheres. There is, therefore, a correlation between the in vitro release and in vivo availability. Interestingly, the peak plasma concentration followed a pattern closely similar to the AUC and this provided a further index of the bioavailability of the drug studied. The generally high AUC values obtained for the microspheres may be an indication that the absorption of cefuroxime sodium was both rapid and complete and that the drug may have bypassed the hepatic first-pass metabolism. It is noteworthy to point out that adequate precautions were taken to deposit the encapsulated microspheres on the lower or middle part of the rat's rectum with the expectation of causing the absorbed drug to drain directly into the general circulation via either the lower or middle haemorrhoidal veins. This expectation may have been realized considering the high AUC values obtained for the microspheres. It is also deducible from Fig. 4 that the bioavailability as well as the time to attain peak peak plasma level for cefuroxime sodium was generally higher from the microspheres in comparison with that of the control.

\section{CONCLUSION}

The results have shown that the entrapment of cefuroxime sodium in gelatin-mucin microspheres can be used to improve the rectal delivery of the drug. Thus, the findings may be exploited in the design of a rectal delivery system for the drug.

\section{ACKNOWLEDGEMENT}

The technical assistance by Dr. John I. Ihedioha of the Department of Veterinary Pathology and Microbiology of our University in the pharmacokinetic studies is gratefully and deeply acknowledged.

\section{REFERENCES}

1. De Blaey CJ, Tukker JJ. Suppositories and Pessaries. In: Aulton ME (eds). Pharmaceutics: The Science of Dosage Form Design, ed 3 Leicester,UK, Churchill Livingstone, 1988, pp 412-414.

2. Rossi S, Sandri G, Ferrari F, Bonferoni MC, Caramells C. Development of films and matrices based on chitosan and polyacrylic acid for vaginal delivery of acyclovir. STP Pharma Sciences 2003; 13: 183198.

3. Illum L, Wright J, Davis SS. Targeting of microspheres to sites of inflammation. Int J Pharm 1989; 52: 221 224.

4. Davis SS, Illum L. The targeting of Drugs Using Polymeric Microspheres. In: Davis SS, Illum L (eds). Polymeric Drug Delivery System, ed 2, Nothingham, UK, University of Nothingham Press, 1983, pp 1-113. 
5. Coombes A, Tasker S, Lindblal M, Holmgreen $K$, Hoste V. Biodegradable polymeric microparticles for drug delivery and vaccine formulation: The surface attachment of hydrophilic species using the concept of poly(ethylene)glycol achoring segments. Biomaterials 1997; 18: 1153-1161.

6. Ida G, Monica C, Annalia A, Bice C, Luisa M. Influence of glutaraldehyde on drug release and mucoadhesive properties of chitosan microspheres. Carbohyd Polym Sci 1998; 42: 8188.

7. Albertsson AC, Carlfors J, Sturesson C. Preparation and characterization of poly (adipic anhydride) microspheres for ocular drug delivery. J Appl Polym Sci 1995; 62: 695-705.

8. Kedziericz F, Lombry C, Rios R, Hoffman PM. Effect of the formulation on the in vitro release of propranolol from gellan beads. Int J Pharm.1999; 178: 129-136.

9. Santucci E, Alhaique F, Carafa M.,.Coviello T. Gellan for the formulation of sustained delivery beads. J Control Rel 1996; 42: 157-164.

10. Gupta KC, Ravi-Kumar MNV. Drug release behaviour of beads and microgranules of chitosan. Biomaterials 2000; 21: 1115-1119.

11. Gupta KC, Ravi-Kumar MNV. Semi-interpenetrating polymer network beads of crosslinked chitosanglycine for controlled release of chlorpheniramine maleate. J Appl Polym Sci 2000; 76: 672-683.

12. Wakiyama NJK, Nakano M.. Preparation and evaluation of in vitro and in vivo polylactic acid microspheres containing dibucaine. Chem. Pharm. Bull 1982; 30: 3719-3727.
13. Alpar HO, Field WN, Hyde R, Lewis DA. The transport of microspheres from the gastrointestinal tract to inflammatory air pouches in the rat. $\mathrm{J}$ Pharm Pharmacol 1989; 41: 194-196.

14. Yin $Y, X u M$, Chen $X$, Yao $K O$. Drug release behaviour of chitosan/gelatin network polymer microspheres. Chinese Sci Bull 1996; 41: 1266-1270.

15. Mortazari SA, Carpenter BG, Smart JD. A comparative study on the role played by mucus glycoproteins in the rheological behaviour of the mucoadhesive/mucosal interface. Int $\mathrm{J}$ Pharm 1993: 94: 195-207.

16. Stone SH. Methods for obtaining venous blood from the orbital sinus of a rat or mouse. Science 1954 119: 100-102.

17. Riley V. Adaptation of orbital bleeding technique to rapid serial blood studies. Proceedings of the Society for Experimental Biology and Medicine 1950; 104: 751-755.

18. Tietz NW. Preparation of Protein-free Filtrate.In : Tietz NW (eds). Fundamentals of Clinical Chemistry ed 2, Philadelphia, USA, W. B. Saunders Company, 1970, pp 32-45

19. Higuchi T. Mechanism of sustained action medication: theoretical analysis of rate of release of solid drugs dispersed in solid matrices. J Pharm Sci 1963; 52: 1145-1149.

20. Peppas NA.Analysis of Fickian and non-Fickian drug release from polymers. Pharm Acta Helv 1985; 60: 110-111.

21. Philip JC, Karrar AK, Dale LM, Jomjai S. Development and evaluation of a multiple-unit oral sustained release dosage form for $\mathrm{S}(+)$-ibuprofen:preparation and release kinetics.Int J Pharm 1999; 197:73-84. 